\title{
Магнитно-резонансная томография
}

\section{в режиме динамического контрастирования в дифференциальной диагностике глиальных опухолей головного мозга}

\author{
Нечипай Э.А. ${ }^{\text {* }}$, Долгушин М.Б. ${ }^{1}$, Пронин А.И. ${ }^{1}$, Кобякова Е.А. ${ }^{1}$, Фадеева Л.М. ${ }^{2}$ \\ 1 ФГБУ “Национальный медицинский исследовательский центр онкологии им. Н.Н. Блохина” Минздрава России, \\ Москва, Россия \\ 2 ФГАУ “Национальный медицинский исследовательский центр нейрохирургии им. акад. Н.Н. Бурденко” \\ Минздрава России, Москва, Россия
}

\section{Dynamic Contrast Enhanced MRI in Glioma Diagnosis}

\author{
Nechipay E.A. ${ }^{*}$, Dolgushin M.B. ${ }^{1}$, Pronin A.I. ${ }^{1}$, Kobyakova E.A. ${ }^{1}$, Fadeeva L.M. ${ }^{2}$
}

${ }^{1}$ N.N. Blokhin National Medical Research Center of Oncology, Moscow, Russia

${ }^{2}$ N.N. Burdenko National Medical Research Center of Neurosurgery, Moscow, Russia

Цель исследования: изучение возможности применения методики магнитно-резонансного динамического контрастирования (МР-ДК) в уточняющей диагностике глиальных опухолей головного мозга и дифференциации их между собой по признаку степени злокачественности. В связи с этим авторами оценена эффективность ряда перфузионных параметров ( $\mathrm{K}^{\text {trans }}, \mathrm{K}_{\mathrm{ep}}, \mathrm{V}_{\mathrm{e}}$ и iAUC) в решении этой задачи.

Материал и методы. В исследование включены наблюдения 54 пациентов с установленным наличием глиальных новообразований вещества головного мозга. Глиомы Grade I-II диагностированы в $13(24,1 \%)$, а глиомы Grade III-IV - в 41 (75,9\%) случае. Морфологическая верификация диагноза по образцам опухолевой ткани, полученной в результате либо хирургического удаления опухоли, либо путем стереотаксической биопсии, была достигнута у $31(57,4 \%)$ пациента: глиальные опухоли Grade I-II идентифицированы у $6(19,4 \%)$, а глиомы Grade III-IV - у 25 (80,6\%) пациентов.

Результаты. По данным МР-ДК с увеличением степени злокачественности глиальной опухоли отмечается повышение всех исследуемых перфузионных параметров: так, наиболее низкие значения $\mathrm{K}^{\text {trans }}, \mathrm{K}_{\mathrm{ep}}, \mathrm{V}_{\mathrm{e}}$ и iAUC выявлены в глиомах низкой степени злокачественности $\left(0,026\right.$ мин $^{-1}, 0,845$ мин $^{-1}, 0,024$ и 1,757 соответственно), наиболее высокие - в глиомах Grade III-IV (0,052 мин ${ }^{-1}$, 1,083 мин ${ }^{-1}, 0,06$ и 2,694 соответственно). Наиболее информативными показателями (с чувствительностью и специфичностью 90 и 100\% соответственно) в диффе- ренциальной диагностике глиом Grade I-II от Grade III-IV являются $\mathrm{K}^{\text {trans }}$ (cut-off $=0,16 \mathrm{мин}^{-1}$ ) и $\mathrm{V}_{\mathrm{e}}$ (cut-off $=0,13$ ).

Заключение. Методику МР-ДК можно применять в целях дифференциальной диагностики глиальных опухолей разной степени злокачественности.

Ключевые слова: динамическое контрастирование, МР-перфузия, глиальные опухоли, головной мозг.

Ссылка для цитирования: Нечипай Э.А., Долгушин М.Б., Пронин А.И., Кобякова Е.А., Фадеева Л.М. Магнитно-резонансная томография в режиме динамического контрастирования в дифференциальной диагностике глиальных опухолей головного мозга. Медицинская визуализация. 2017; 21 (4): 88-96. DOI: 10.24835/1607-0763-2017-4-88-96.

$$
\star \star *
$$

The aim: to examine the possibility of using dynamic contrast enhanced magnetic resonance imaging (DCE MRI) in clarifying the diagnosis of glial brain tumors and the differentiation between them on the basis of the malignancy degree. In this regard, the authors evaluated the effectiveness of perfusion parameters ( $\mathrm{K}^{\text {trans }}, \mathrm{K}_{\mathrm{ep}}, \mathrm{V}_{\mathrm{e}}$ and $\mathrm{iAUC}$ ).

Materials and methods. The study included examination of 54 patients with an established presence of brain glial tumors. Glioma Grade I-II diagnosed in 13 (24.1\%) and glioma Grade III-IV in 41 (75.9\%) cases. Morphological verification of the diagnosis obtained as a result of either surgical removal of the tumor or stereotactic biopsy was achieved in 
$31(57.4 \%)$ patients: glial tumors Grade I-II identified in 6 (19.4\%), and glioma Grade III-IV - 25 (80.6\%) cases.

Results. According to DCE increasing of the malignancy degree of glial tumors is followed by increasing of all perfusion parameters: thus, the lowest values of $\mathrm{K}^{\text {trans }}, \mathrm{K}_{\mathrm{ep}}, \mathrm{V}_{\mathrm{e}}$ and iAUC were identified in low grade gliomas $\left(0.026 \mathrm{~min}^{-1}\right.$, $0.845 \mathrm{~min}^{-1}, 0.024$ and 1.757 , respectively), the highest in gliomas Grade III-IV (0.052 $\mathrm{min}^{-1} 1.083 \mathrm{~min}^{-1}, 0.06$ and 2.694, respectively). The most informative parameters with sensitivity $90 \%$ and specificity $100 \%$ in the differential diagnosis of gliomas Grade I-II and Grade III-IV are K ${ }^{\text {trans }}$ (cut-off = $0.16 \mathrm{~min}^{-1}$ ) and $V_{e}$ (cut-off $=0.13$ ).

Conclusion. DCE MRI can be used in differential diagnosis of glial brain tumors of different malignancy grade.

Key words: dynamic contrast enhanced, MR-perfusion, glial tumors, brain.

Recommended citation: Nechipay E.A., Dolgushin M.B., Pronin A.I., Kobyakova E.A., Fadeeva L.M. Dynamic Contrast Enhanced MRI in Glioma Diagnosis. Medical visualization. 2017 ; 21 (4): 88-96.

DOI: 10.24835/1607-0763-2017-4-88-96.

$$
\star * *
$$

\section{Введение}

Гематоэнцефалический барьер (ГЭБ) - физиологический барьер между кровеносной и центральной нервной системой (ЦНС), главная функция которого - поддержание гомеостаза мозга в норме обеспечивается отсутствующими в сосудах других анатомических частей тела человека защитными "механизмами". Особенностью церебральных капилляров является наличие в их стенке эндотелиоцитов, базальной мембраны, а также перицитов и астроцитов, отсутствующих в сосудах других тканей организма (эндотелий сосудов большинства тканей содержит фенестрации и межклеточные щели, через которые вода и растворенные вещества проникают из сосудов во внесосудистые интерстициальные пространства и обратно). Контакты между клетками, формирующими капилляры головного мозга, более плотные, между клетками практически отсутствуют фенестрации и межклеточные щели, в результате эндотелиальная выстилка капилляров мозга является сплошной [1]. Все перечисленное предотвращает поступление из сосудов головного мозга во внесосудистое интерстициальное пространство ряда веществ, в частности таких, как экзогенные MPконтрастные вещества (КВ).

Известно, что для большинства опухолей ЦНС характерны различные «паттерны» накопления КВ, которые свидетельствуют в пользу наличия в них участков нарушенного ГЭБ. Нарушение ГЭБ связано с развитием в опухоли патологической сосудистой сети, которая появляется уже при достижении опухоли размеров более 2-4 мм $^{3}$ [2]. Дальнейший рост и развитие опухоли увеличивают потребность в поступлении питательных веществ в патологическую ткань. Для обеспечения этого процесса опухолевые клетки под влиянием гипоксии и гипогликемии начинают секретировать цитокины, стимулирующие рост новых патологических сосудов (неоангиогенез), что является характерной особенностью злокачественных новообразований [3]. В развитии неоангиогенеза главенствующая роль отводится эндотелиальному фактору роста сосудов (VEGF), который запускает механизм развития последних из уже имеющихся, увеличивает микроваскулярную проницаемость стенки без нарушения целостности эндотелиоцитов и вызывает вазодилатацию неизмененных сосудов [4-8]. Образованные в результате неоангиогенеза опухолевые сосуды характеризуются повышенной извитостью, наличием множества артериовенозных шунтов, выраженной хрупкостью стенки, что в свою очередь приводит к несостоятельности ГЭБ и свободной экстравазации КВ через сосудистую стенку в интерстициальное пространство [9].

\footnotetext{
Для корреспонденции*: Нечипай Эмилия Андреевна -115478 Москва, Каширское шоссе, д. 24, ФГБУ “НМИЦ онкологии им. Н.Н. Блохина" МЗ РФ. Тел.: +7-967-121-80-50. E-mail: nechipay@hotmail.com

Нечипай Эмилия Андреевна - аспирант рентгенодиагностического отделения отдела лучевой диагностики и интервенционной радиологии НИИ КиЭР ФГБУ “НМИЦ онкологии им. Н.Н. Блохина" МЗ РФ, Москва; Долгушин Михаил Борисович - доктор мед. наук, заведующий отделением позитронно-эмиссионной томографии отдела лучевой диагностики и интервенционной радиологии НИИ КиЭР ФГБУ "НМИЦ онкологии им. Н.Н. Блохина" МЗ РФ, Москва; Пронин Артем Игоревич - научный сотрудник отделения позитронной эмиссионной томографии НИИ КиЭР ФГБУ "НМИЦ онкологии им. Н.Н. Блохина" МЗ РФ, Москва; Кобякова Екатерина Алексеевна младший научный сотрудник рентгенодиагностического отделения отдела лучевой диагностики и интервенционной радиологии НИИ КиЭР ФГБУ "НМИЦ онкологии им. Н.Н. Блохина" МЗ РФ, Москва; Фадеева Людмила Михайловна - ведущий инженер отделения рентгенохирургических методов диагностики и лечения ФГАУ “НМИЦ нейрохирургии им. акад. Н.Н. Бурденко” МЗ РФ, Москва.
}

Contact*: Emiliya A. Nechipay - 115478, Moscow, Kashirskoe shosse, 23, N.N. Blokhin NMRCO. Phone: 8 (967) 121-80-50.

E-mail: nechipay@hotmail.com

Emiliya A. Nechipay - PhD-student of Division of Diagnostic Radiology and Interventional Radiology of N.N. Blokhin NMRCO, Moscow; Mikhail B. Dolgushin - doct. of med. sci., head of PET department of N.N. Blokhin NMRCO, Moscow; Artem I. Pronin - reseach fellow of PET/CT department of N.N. Blokhin NMRCO, Moscow; Ekaterina A. Kobyakova - medical doctor of Division of Diagnostic Radiology and Interventional Radiology of N.N. Blokhin NMRCO, Moscow; Ludmila M. Fadeeva - engineer of Radiology and surgery methods of diagnostic and treatment Department of N.N. Burdenko NMRCO, Moscow. 
Для определения биологической активности и степени злокачественности глиальной опухоли, таким образом, достаточно производить оценку выраженности неоангиогенеза. По данным K.H. Plate и соавт. (1992) [10] и S. Erdamar и соавт. (2006) [11], экспрессия VEGF непосредственно связана со степенью злокачественности глиальных опухолей.

С внедрением в схемы лечения пациентов с глиальными новообразованиями головного мозга антиангиогенных препаратов, подавляющих формирование патологических сосудов, возникла потребность в изучении механизмов неоангиогенеза для дальнейшей оценки эффективности противоопухолевой терапии. В качестве инструмента оценки опухолевого неоангиогенеза в мировой практике набирает популярность методика магнитно-резонансного динамического контрастирования (МР-ДК) [12]. Так, Н.P. Schlemmer и соавт. [13] и Н. Hawighorst и соавт. [14, 15] использовали данный метод для выявления специфических характеристик опухолей, а P. Padhani и соавт. [16] и A.L. Thomas и соавт. [17] изучали васкулярное ремоделирование после проведенного противоопухолевого лечения.

\section{Цель исследования}

Изучение показателей перфузионных параметров $\mathrm{K}^{\text {trans }}, \mathrm{K}_{\mathrm{ep}}, \mathrm{V}_{\mathrm{e}}$ и iAUC в солидных компонентах глиальных опухолей низкой и высокой степени злокачественности с оценкой возможности применения методики МР-ДК для проведения дифференциальной диагностики между такими опухолями.

\section{Материал и методы}

В исследование включены клинические наблюдения 54 пациентов (мужчин - 30 (55,6\%), женщин - $24(44,4 \%))$, страдающих глиальными опухолями головного мозга разной степени злокачественности: глиомами Grade I-II - 13 (24,1\%) и глиомами Grade III-IV- 41 (75,9\%). Морфологическая верификация диагноза по образцам опухолевой ткани, полученной либо в ходе хирургического удаления опухоли, либо путем стереотаксической биопсии (СТБ), была достигнута у 31 (57,4\%) пациента: глиальные опухоли Grade I-II - 6 (19,4\%) и глиомы Grade III-IV - 25 (80,6\%). У 23 (42,6\%) пациентов хирургические вмешательства, направленные на получение образцов опухолевой ткани, не выполнялись в связи с: а) содержанием стандартов лечения; б) тяжелым соматическим статусом и высоким операционно-анестезиологическим риском; в) особенностями локализации опухоли; г) отказом пациента от операции. В подобных случаях постановка диагноза основывалась на данных комплекса диагностических мероприятий, направленных на уточняющую диагностику опухоли и на исключение первичных экстракраниальных поражений с метастазами в головном мозге методами МРТ, КТ и ПЭТ.

МР-исследования выполняли на томографе с напряженностью поля 3,0 Тл (Skyra, Siemens AG). Исследование включало в себя:

а) стандартные последовательности до внутривенного введения КВ - T1-SE (TR/TE = 6600/100), T2-SE (2000/9), T2-FLAIR (9000/81), DWI (7700/98, b-values = 50 и 800);

б) две последовательности T1-VIBE с разными углами отклонения $\left(4,37 / 1,67\right.$; flipangle $2^{\circ}$ и $\left.15^{\circ}\right)$ для получения "нативных" карт Т1-релаксации тканей и МР-ДК последовательности с отсроченным болюсным внутривенным введением КВ;

в) в завершение - T1-VIBE после внутривенного контрастирования $(20 / 1,20)$ с последующей 3D-реконструкцией.

Параметры МР-ДК: ТR 3,12 мс; ТЕ 1,18 мс; flipangle 250; длительность динамики 3,2 c; размер пикселя 0,8 × 0,8 мм; FOV = 260; 36 срезов с толщиной среза 2,5 мм; время сканирования 2 мин 57 c.

Динамическое контрастирование (МР-ДК) проводили с болюсным внутривенным введением стандартного количества KB (Omniscan, GE Healthcare) из расчета 0,1 ммоль/кг массы со скоростью введения 5 мл/с с последующим болюсным введением 20 мл физиологического раствора со скоростью 5 мл/с.

Для качественной оценки морфоструктурных особенностей опухоли использовались стандартные последовательности (Т1ВИ, Т2ВИ, T2-FLAIR, DWI). Для анализа результатов MP-ДК в режиме off line на рабочей станции при помощи программы Tissue 4D количественно оценивали изображения, полученные в режиме TWIST с выбором зон интереса (ROI) в солидной части опухолей (соответствует области гомогенного накопления KB в режиме T1-VIBE) с вычислением перфузионных показателей и построением соответствующих параметрических карт. Кистозные, некротические компоненты, зона геморрагических изменений и проходящие в толще сосуды были исключены из зоны интереса (ROI).

Для анализа данных, полученных при обработке протоколов исследования, применяли методы описательной статистики (среднее, стандартное отклонение, медиана, квантили и т.п.) и ROCанализа. 


\section{Результаты}

Медианы значений показателей $K^{\text {trans }}, V_{\mathrm{e}}$ и iAUC в глиальных опухолях Grade I-II достоверно $(p<0,05)$ превосходили таковые в неизмененном белом веществе головного мозга как на ипси-, так и на контралатеральной стороне и составили:

а) для $\mathrm{K}^{\text {trans }}-0,026$ мин $^{-1}$ против $0,012^{-1}$ и $0,017 \mathrm{Mин}^{-1}$ соответственно;

б) для $V_{e}-0,024$ против 0,012 и 0,015 соответственно;

в) для iAUC - 1,757 против 0,965 и 0,910 соответственно.

Медиана значений $\mathrm{K}_{\text {ep }}$ в глиомах Grade I-II оказалась ниже, чем в неизмененном белом веществе ипси- и контралатеральной стороны головного мозга, что может быть обусловлено задержкой КВ в интерстициальном пространстве опухоли и медленным его выведением обратно в сосудистое русло, в то время как в неизмененном веществе мозга поступление КВ в интерстициальное пространство отсутствует как таковое (табл. 1).

Однако при анализе параметрической карты каждого из параметров выявленное повышение их значений не получило своего отображения на картах (рис. 1).

Как следует из табл. 1, медианы значений Ktrans, $\mathrm{V}_{\mathrm{e}}$ и iAUC в глиальных опухолях высокой степени злокачественности оказались значительно (для $\mathrm{K}^{\text {trans }}$ - двухкратно, для $\mathrm{V}_{\mathrm{e}}$ и iAUC - трехкратно) выше таковых в глиомах низкой степени злокачественности, так, медиана значений $\mathrm{K}^{\text {trans }}$ в глиомах Grade III-IV составила 0,052 мин ${ }^{-1}, V_{\mathrm{e}}$ и iAUC 0,06 и 2,694 соответственно.

На всех параметрических картах в опухолях Grade III-IV отмечались множественные участки повышенного MP-сигнала, что может быть объяснено неоднородностью структуры таких новообразований (рис. 2).

При сравнительной оценке изучаемых перфузионных параметров, в частности их максимальных и минимальных значений, между группами глиальных опухолей Grade I-II и Grade III-IV выявлено, что отсутствуют пересечения диапазонов значений показателей $\mathrm{K}^{\text {trans }}$ и $\mathrm{V}_{\mathrm{e}}$, в то время как диапазоны значений показателей $\mathrm{K}_{\text {ep }}$ в опухолях Grade I-II и Grade III--IV крайне близки друг к другу, а диапазоны значений iAUC в них пересекаются (рис. 3).

Таким образом, определенное количество клинических наблюдений в каждой из сравниваемых групп по уровню значений параметра IAUC и $\mathrm{K}_{\text {ep }}$ (в отличие от параметров $K^{\text {trans }} n V_{e}$ ) не могут быть однозначно отнесены к тому или иному типу глиальной опухоли. Для определения точки разделения (порог cut-off) количественных значений этих параметров и отнесения опухоли либо к низко-, либо к высокозлокачественному типу нами применен ROC-анализ.

Количественную оценку характеристической кривой проводили при расчете площади под ней (Area Under Curve - AUC), для этого применяли рекомендованную шкалу значений AUC (табл. 2).

Наилучшим качеством в дифференциальной диагностике глиальных опухолей Grade I-II от Grade III-IV обладают показатели K ${ }^{\text {trans }}, \mathrm{V}_{\mathrm{e}}$ и iAUC, что свидетельствует о сопоставимой эффективности этих параметров в диагностике данных типов опухолей. Для оценки степени злокачественности глиальных опухолей в табл. 2 отображены пороги cut-off c определенной для них чувствительностью и специфичностью для всех изучаемых перфузионных параметров. Таким образом, наиболее информативными показателями (с чувствительностью и специфичностью 90 и 100\% соответственно) в дифференциальной диагностике глиом Grade I-II от Grade III-IV головного мозга являются $\mathrm{K}^{\text {trans }}$ (cut-off $=0,16$ мин $^{-1}$ ) и $\mathrm{V}_{\mathrm{e}}$ (cut-off $=0,13$ ). Параметр iAUC, несмотря на высокий показатель AUC $(1,00)$, мы не рассматриваем в качестве высокоинформативного из-за его низкой специфичности $(0,11)$.

С увеличением степени злокачественности глиальной опухоли отмечается и повышение показателей $\mathrm{K}^{\text {trans }}$ и V $\mathrm{V}_{\mathrm{e}}$, которые свидетельствуют о возрастании количества поступающего количества КВ во внеклеточное внесосудистое пространство.

Таблица 1. Значения перфузионных параметров в глиальных опухолях Grade I-II и Grade III-IV относительно неизмененного вещества головного мозга $(p<0,05)$

\begin{tabular}{l|c|c|c|c}
\hline \multirow{2}{*}{ Зона интереса } & \multicolumn{3}{|c}{ Исследуемый перфузионный параметр (медиана) } \\
\cline { 2 - 4 } & $\mathrm{K}^{\text {trans }}$, мин $^{-1}$ & $\mathrm{~V}_{\mathrm{e}}$ & $\mathrm{K}_{\mathrm{ep}}$, мин $^{-1}$ & $\mathrm{iAUC}$ \\
\hline Глиальная опухоль Grade I-II & 0,026 & 0,024 & 0,845 & 1,757 \\
Глиальная опухоль Grade III-IV & 0,052 & 0,06 & 1,083 & 2,694 \\
Неизмененное вещество головного мозга & 0,012 & 0,012 & 1,106 & 0,965 \\
$\begin{array}{l}\text { ипсилатеральной стороны } \\
\text { Неизмененное вещество головного мозга } \\
\text { контралатеральной стороны }\end{array}$ & 0,017 & 0,015 & 1,074 & 0,910 \\
\hline
\end{tabular}



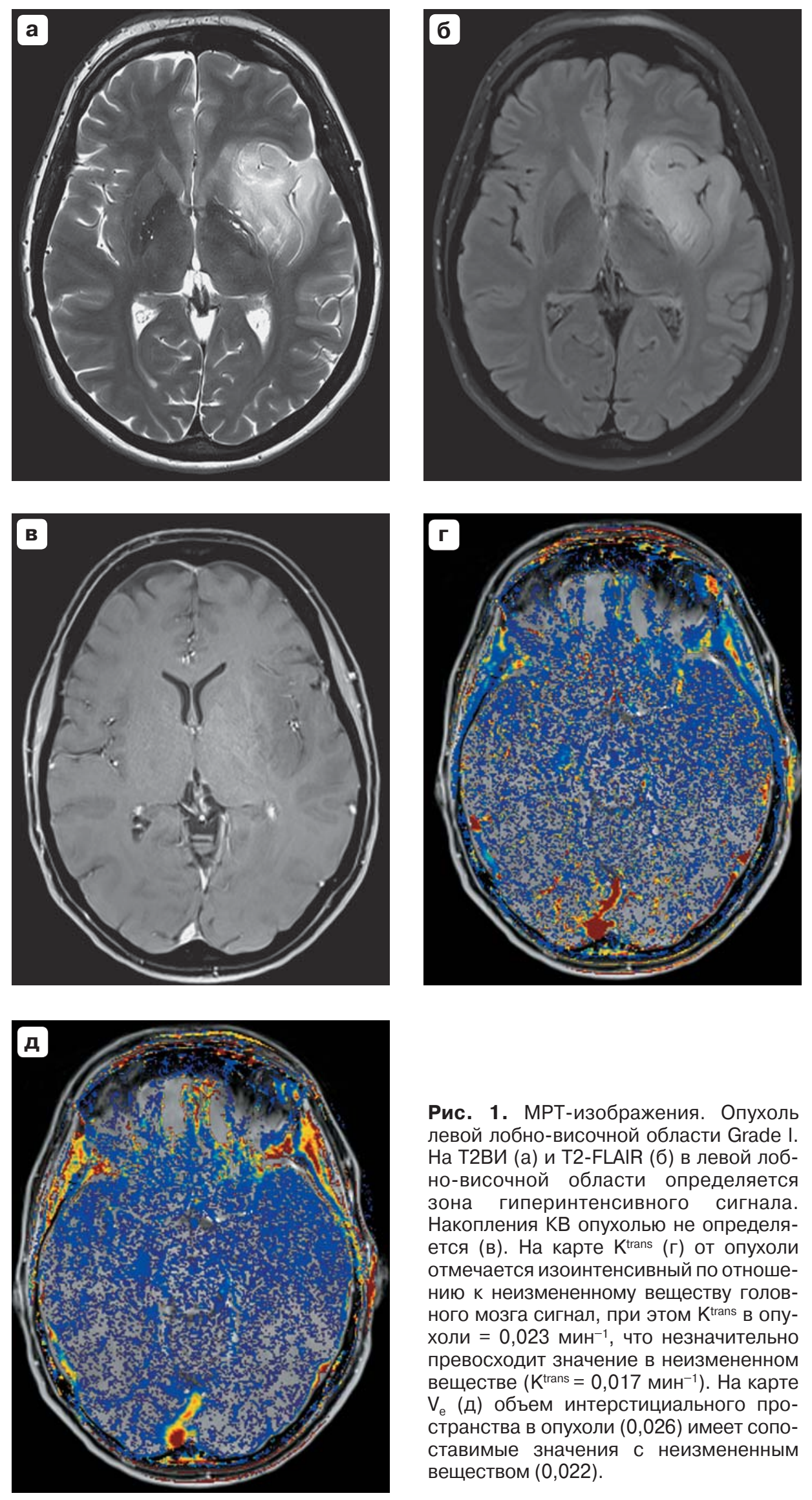

Рис. 1. МРТ-изображения. Опухоль левой лобно-височной области Grade I. На T2ВИ (а) и T2-FLAIR (б) в левой лобно-височной области определяется зона гиперинтенсивного сигнала. Накопления КВ опухолью не определяется (в). На карте $\mathrm{K}^{\text {trans }}$ (г) от опухоли отмечается изоинтенсивный по отношению к неизмененному веществу головного мозга сигнал, при этом $\mathrm{K}^{\text {trans }}$ в опухоли $=0,023$ мин $^{-1}$, что незначительно превосходит значение в неизмененном веществе $\left(\mathrm{K}^{\text {trans }}=0,017\right.$ мин $\left.^{-1}\right)$. На карте $\mathrm{V}_{\text {е }}$ (д) объем интерстициального пространства в опухоли $(0,026)$ имеет сопоставимые значения с неизмененным веществом $(0,022)$. 

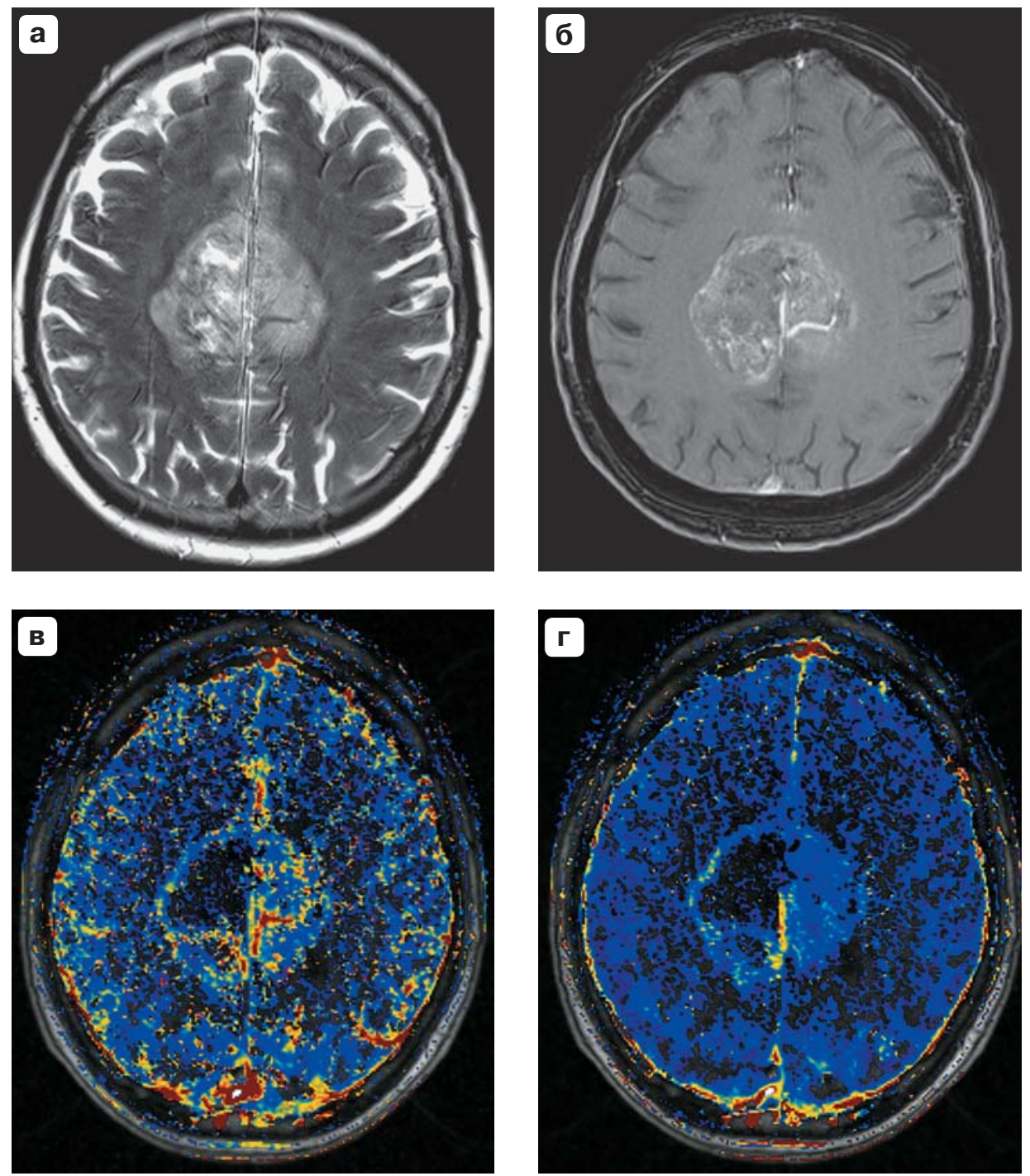

Рис. 2. МРТ-изображения. Опухоль левого полушария головного мозга Grade IV. В левой лобно-затылочной доле образование гетерогенной структуры, гиперинтенсивного сигнала на Т2 (а) с участками некроза. После введения КВ (б) отмечается его неравномерное накопление солидной частью опухоли.. На карте $\mathrm{K}^{\text {trans }}(\mathrm{B})$ - множественные участки повышенной проницаемости $\left(\mathrm{K}^{\text {trans }}=0,078 \mathrm{мин}^{-1}\right)$ и объема интерстициального пространства $\left(\mathrm{V}_{\mathrm{e}}=0,37\right)$ на карте $\mathrm{V}_{\mathrm{e}}(г)$. 
a

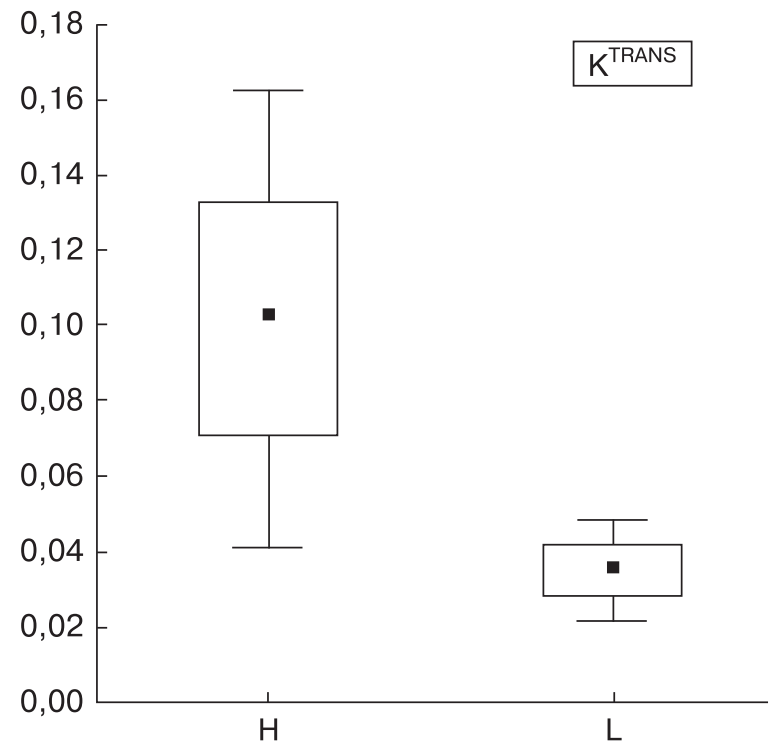

B

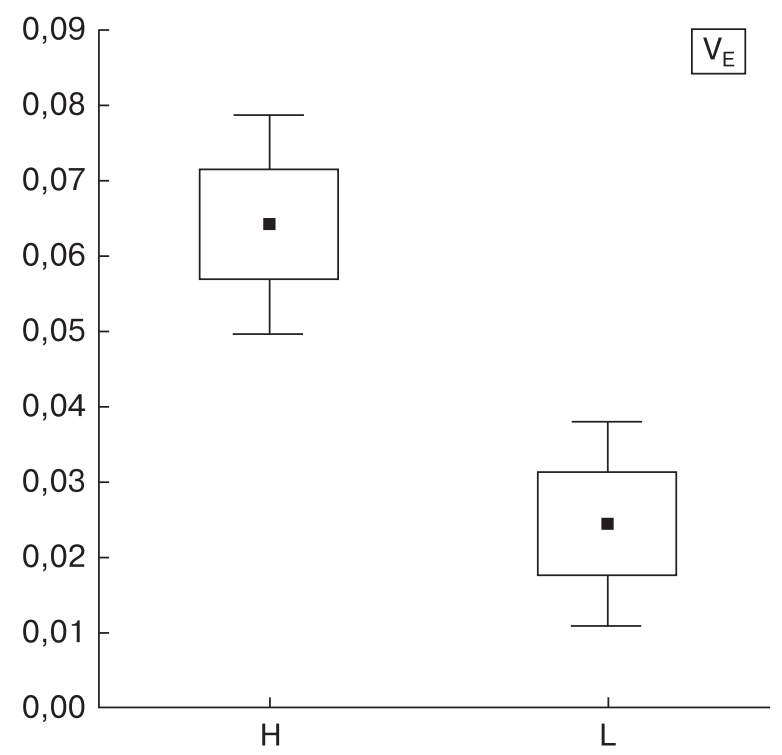

6

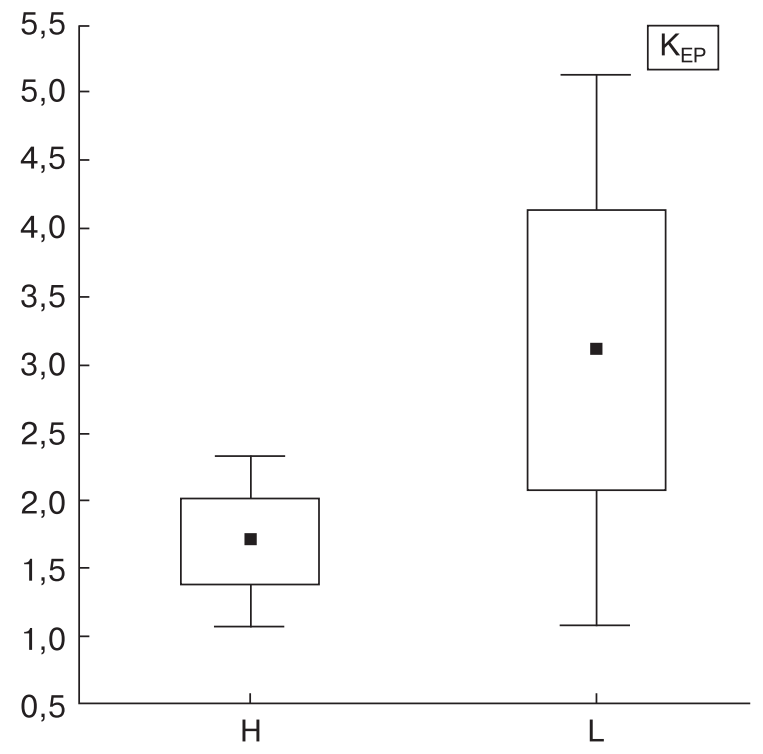

$\mathbf{\Gamma}$

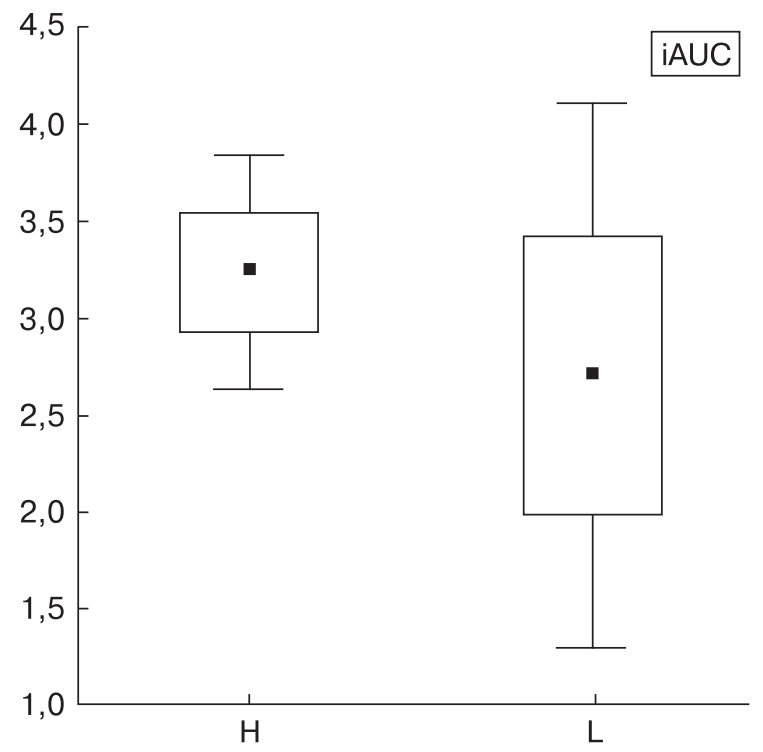

Рис. 3. Диапазоны минимальных и максимальных количественных значений параметров перфузии $\mathrm{K}^{\text {trans }}, \mathrm{K}_{\mathrm{ep}}, \mathrm{V}_{\mathrm{e}}$ и iAUC в группах глиальных опухолей Grade I-II и Grade III-IV (гистограмма): пересечение диапазонов отмечено только для iAUC.

Таблица 2. Результаты ROC-анализа для дифференцирования глиальных опухолей Grade I-II Oт Grade III-IV (на основе данных таблиц координат ROC-кривой)

\begin{tabular}{l|c|c|c|c|c}
\hline \multirow{2}{*}{\multicolumn{1}{c|}{ Показатель }} & \multicolumn{5}{|c}{ Перфузионные параметры } \\
\cline { 2 - 5 } & $\mathrm{K}^{\text {trans }}$ очаг & $\mathrm{V}_{\text {е очаг }}$ & $\mathrm{K}_{\text {ер очаг }}$ & iAUC & $\mathrm{V}_{\text {еконтр сторона }}$ \\
\hline AUC & $\mathbf{0 , 9 0}$ & $\mathbf{0 , 9 1}$ & 0,63 & $\mathbf{1 , 0 0}$ & 0,79 \\
Порог cut-оff & 0,016 & 0,013 & 1,25 & 8,79 & 0,02 \\
Специфичность & $\mathbf{1 , 0 0}$ & $\mathbf{1 , 0 0}$ & 0,00 & 0,11 & 1,00 \\
Чувствительность & $\mathbf{0 , 9 0}$ & $\mathbf{0 , 9 0}$ & 0,63 & $\mathbf{1 , 0 0}$ & 0,79 \\
\hline
\end{tabular}


Оба параметра ( $\mathrm{K}^{\text {trans }}$ и $\mathrm{V}_{\mathrm{e}}$ ) могут быть использованы для проведения дифференциальной диагностики между глиальными опухолями Grade I-II и Grade III-IV.

\section{Обсуждение}

Наиболее низкие значения $K^{\text {trans }}\left(0,026\right.$ мин $\left.^{-1}\right)$ выявлены в глиальных опухолях Grade I-II, а с увеличением степени злокачественности отмечалось повышение данных показателей в глиомах Grade III-IV (0,052 мин $\left.{ }^{-1}\right)$. Результаты нашего исследования согласуются с данными H.C. Roberts и соавт. (2000), которые также выявили хорошую корреляцию между значением $\mathrm{K}^{\text {trans }}$ и степенью злокачественности глиом, что может быть объяснено постепенным возрастанием метаболических потребностей в опухоли, ведущих к тканевой гипоксии, которая в свою очередь запускает механизм развития неоангиогенеза [18]. А с увеличением количества патологических сосудов в новообразованиях, характеризующихся извитой и хрупкой стенкой, возрастает и количество КВ, проникающего через поврежденный ГЭБ во внеклеточное внесосудистое пространство. Так, по нашим данным, с увеличением степени злокачественности глиальных опухолей отмечается повышение значений $\mathrm{K}^{\text {trans }}$, что совпадает с результатами H.C. Roberts и соавт. (2000) [18], впервые описавшими зависимость между $\mathrm{K}^{\text {trans }}$ и степенью злокачественности глиом, а также H.C. Roberts и соавт. (2001) [19] и Т.A. Patankar и соавт. (2005) [20], пришедшими к выводу о том, что глиальные опухоли Grade III-IV имеют более высокие значения $\mathrm{K}^{\text {trans }}$ по сравнению с глиомами Grade I-II, а также с результатами H.S. Choi и соавт. (2013) [21]. Таким образом, параметр $\mathrm{K}^{\text {trans }}$ предоставляет важную диагностическую информацию о процессах, протекающих в опухолевой ткани.

Медиана значений $\mathrm{V}_{\text {е }}$ в нашем клиническом материале также повышалась в направлении от глиальных опухолей Grade I-II до опухолей Grade III-IV и составила для первых 0,024, а для вторых - 0,06. Показатель $\mathrm{V}_{\mathrm{e}}$, как и $\mathrm{K}^{\mathrm{trans}}$, коррелирует со степенью злокачественности глиальных опухолей [22, 23]. Значение объема интерстициального пространства в дифференциальной диагностике глиом разной степени злокачественности между собой остается малоизученным, известно лишь небольшое количество работ, посвященных данному вопросу [24, 25]. При сравнении наших результатов с данными этих исследователей, несмотря на различия в цифровых значениях этого перфузионного параметра, отмечалась аналогичная тенденция к увеличению значений $\mathrm{V}_{\mathrm{e}}$ по мере повышения степени злокачественности глиальной опухоли. Повышение фракционного интерстициального объема в высокозлокачественных опухолях также подтверждает потенциальное использование перфузионных параметров, характеризующих состояние интерстициального пространства в дифференциальной диагностике опухолей ЦНС [26]. Считается, что интерстициальное пространство соотносится с индексом опухолевого некроза и обратным индексом опухолевой целлюлярности [27], что может быть обусловлено быстрым ростом опухоли, ведущим к регионарной гипоксии и формированию зон некрозов.

Полученные результаты о $\mathrm{K}^{\text {trans }}$ и $\mathrm{V}_{\mathrm{e}}$ как о наиболее чувствительных и специфичных показателях МР-ДК в дифференциальной диагностике глиом разной степени злокачественности совпали с оценками этих параметров в исследовании N. Zhang и соавт. (2012). Так, ими было показано, что по показателям $\mathrm{K}^{\text {trans }}$ и $\mathrm{V}_{\mathrm{e}}$ можно провести диагностику не только между низко- и злокачественными глиомами, но и отличить Grade II от III [28].

\section{Заключение}

Исследуемые с помощью методики МР-ДК перфузионные показатели микроваскулярной проницаемости ( $\left.\mathrm{K}^{\text {trans }}\right)$ сосудов опухолей вещества головного мозга и объема интерстициального пространства $\left(\mathrm{V}_{\mathrm{e}}\right)$ опухоли можно применять в целях дифференциальной диагностики глиальных опухолей разной степени злокачественности. По нашим данным, для проведения дифференциации глиальных опухолей Grade I-II от Grade III-IV наиболее специфичными показателями являются $\mathrm{K}^{\text {trans }}$ и $\mathrm{V}_{\text {е }}$ (чувствительность 90\%, специфичность $100 \%)$.

\section{Список литературы / References}

1. Pavelka M., Roth J. Funktionelle Ultrastruktur. Verlag Springer, 2009: 234-235.

2. Leenders W., Kusters B., Pikkemaat J., Wesseling P., Ruiter D., Heerschap A., Barentsz J., de Waal R.M. Vascular endothelial growth factor-A determines detectability of experimental melanoma brain metastasis in GD-DTPAenhanced MRI. Int. J. Cancer. 2003; 105 (4): 437-443. DOI 10.1002/ijc. 11102 .

3. Thompson G., Mills S., Coope D., O'Connor J.P., Jackson A. Imaging biomarkers of angiogenesis and the microvascular environment in cerebral tumours. $\mathrm{Br}$. J. Radiol. 2011; 84 Spec No 2: S127-S144.

DOI: $10.1259 / \mathrm{bjr} / 66316279$.

4. Senger D.R., Van deWater L., Brown L.F., Nagy J.A., Yeo K.T., Yeo T.K., Berse B., Jackman R.W., Dvorak A.M., Dvorak H.F. Vascular permeability factor (VPF, VEGF) in tumor biology. Cancer Metastasis Rev. 1993; 12: 303-324.

5. Ashrafpour H., Huang N., Neligan P.C., Forrest C.R., Addison P.D., Moses M.A., Levine R.H., Pang C.Y. Vasodilator effect and mechanism of action of vascular 
endothelial growth factor in skin vasculature. Am. J. Physiol. Heart. Circ. Physiol. 2004; 286 (3): 946-954. DOI: 10.1152/ajpheart.00901.2003.

6. Ku D.D., Zaleski J.K., Liu S.Brock T.A. Vascular endothelial growth factor induces EDRF-dependent relaxation in coronary arteries. Am. J. Physiol. 1993; 265 (2, Pt 2): 586-592.

7. Wei W., Chen Z.W., Yang Q., Jin H., Furnary A., Yao X.Q., Yim A.P., He G.W. Vasorelaxation induced by vascular endothelial growth factor in the human internal mammary artery and radial artery. Vasc. Pharmacol. 2007; 46 (4): 253-259. DOI: 10.1016/j.vph.2006.10.009.

8. Senger D.R., Galli S.J., Dvorak A.M., Perruzzi C.A., Harvey V.S., Dvorak H.F. Tumor cells secrete a vascular permeability factor that promotes accumulation of ascites fluid. Science. 1983; 219 (4587): 983-985.

DOI: $10.1126 /$ science.6823562.

9. Byrne T., Cascino T., Posner J. Brain metastasis from melanoma. J. Neurooncol. 1983; 1 (4): 313-317.

10. Plate K.H., Breier G., Weich H.A., Risau W. Vascular endothelial growth factor is a potential tumour angiogenesis factor in human gliomas in vivo. Nature. 1992; 359 (6398): 845-848.

11. Erdamar S., Bagci P., Oz B., Dirican A. Correlation of endothelial nitric oxide synthase and vascular endothelial growth factor expression with malignancy in patients with astrocytic tumors. J. Buon. 2006; 11 (2): 213-216.

12. Li X., Zhu Y., Kang H., Zhang Y., Liang H., Wang S., Zhang W. Glioma grading by microvascular permeability parameters derived from dynamic contrast-enhanced MRI and intratumoral susceptibility signal on susceptibility weighted imaging. Cancer Imaging. 2015; 15 (1): 4. DOI: 10.1186/s40644-015-0039-z.

13. Schlemmer H.P., Merkle J., Grobholz R., Jaeger T., Michel M.S., Werner A., Rabe J., van Kaick G. Can preoperative contrast-enhanced dynamic MR imaging for prostate cancer predict microvessel density in prostatectomy specimens? Eur. Radiol. 2004; 14 (2): 309-317. DOI: $10.1007 / \mathrm{s} 00330-003-2025-2$.

14. Hawighorst H., Weikel W., Knapstein P.G., Knopp M.V., Zuna I., Schönberg S.O., Vaupel P., van Kaick G. Angiogenic activity of cervical carcinoma: assessment by functional magnetic resonance imaging-based parameters and a histomorphological approach in correlation with disease outcome. Clin. Cancer Res. 1998; 4 (10): 2305-2312.

15. Hawighorst H., Knapstein P.G., Knopp M.V., Weikel W., Brix G., Zuna I., Schönberg S.O., Essig M., Vaupel P., van Kaick G.Uterine cervical carcinoma: comparison of standard and pharmacokinetic analysis of time intensity curves for assessment of tumor angiogenesis and patient survival. Cancer Res. 1998; 58 (16): 3598-3602.

16. Padhani A.R. MRI for assessing antivascular cancer treatments. Br. J. Radiol. 2003; 76, Spec No1: 60-80. https://doi.org/10.1259/bjr/15334380.

17. Thomas A.L., Morgan B., Drevs J., Unger C., Wiedenmann B., Vanhoefer U., Laurent D., Dugan M., Steward W.P. Vascular endothelial growth factor receptor tyrosine kinase inhibitors: PTK787/ZK 222584. Semin. Oncol.
2003; 30 (3, Suppl. 6): 32-38.

DOI: $10.1016 / S 0093-7754(03) 70023-2$.

18. Roberts H.C., Roberts T.P., Brasch R.C., Dillon W.P. Quantitative measurement of microvascular permeability in human brain tumors achieved using dynamic contrastenhanced MR imaging: correlation with histologic grade. Am. J. Neuroradiol. 2000; 21 (5): 891-899.

19. Roberts H.C., Roberts T.P., Bollen A.W, Ley S., Brasch R.C., Dillon W.P. Correlation of microvascular permeability derived from dynamic contrast-enhanced MR imaging with histologic grade and tumor labeling index: a study in human brain tumors. Acad. Radiol. 2001; 8 (5): 384-391. DOI: http://dx.doi.org/10.1016/S1076-6332(03)80545-7.

20. Patankar T.F., Haroon H.A., Mills S.J., Balériaux D., Buckley D.L., Parker G.J., Jackson A. Is volume transfer coefficient (Ktrans) related to histologic grade in human gliomas? Am. J. Neuroradiol. 2005; 26 (10): 2455-2465.

21. Choi H.S., Kim A.H., Ahn S.S., Shin N., Kim J., Lee S-K. Glioma Grading Capability: Comparisons among Parameters from Dynamic Contrast-Enhanced MRI and ADC Value on DWI. Korean J. Radiol. 2013; 14 (3): 487-492. DOI: $10.3348 /$ kjr.2013.14.3.487.

22. Awasthi R., Rathore R.K., Soni P., Sahoo P., Awasthi A., Husain N., Behari S., Singh R.K., Pandey C.M., Gupta R.K. Discriminant analysis to classify glioma grading using dynamic contrast-enhanced MRI and immunohistochemical markers. Neuroradiology. 2012; 54 (3): 205-213. DOI: 10.1007/s00234-011-0874-y

23. Zhang N., Zhang L., Qiu B., Meng L., Wang X., Hou B.L. Correlation of volume transfer coefficient Ktrans with histopathologic grades of gliomas. J. Magn. Reson. Imaging. 2012; 36 (2): 355-363. DOI: 10.1002/jmri.23675.

24. Jia Z., Geng D., Xie T., Zhang J., Liu Y. Quantitative analysis of neovascular permeability in glioma by dynamic contrast-enhanced MR imaging. J. Clin. Neurosci. 2012; 19 (6): 820-823. DOI: 10.1016/j.jocn.2011.08.030.

25. Awasthi R., Rathore R.K., Soni P., Sahoo P., Awasthi A., Husain N., Behari S., Singh R.K., Pandey C.M., Gupta R.K. Discriminant analysis to classify glioma grading using dynamic contrast-enhanced MRI and immunohistochemical markers. Neuroradiology. 2012; 54 (3): 205-213. DOI: 10.1007/s00234-011-0874-y.

26. Lüdemann L., Grieger W., Wurm R., Budzisch M., Hamm B., Zimmer C. Comparison of dynamic contrast-enhanced MRI with WHO tumor grading for gliomas. Eur. Radiol. 2001; 11 (7): 1231-1241. DOI: 10.1007/s003300000748.

27. Mills S.J., Soh C., Rose C.J., Cheung S., Zhao S., Parker G.J., Jackson A. Candidate biomarkers of extravascular extracellular space: a direct comparison of apparent diffusion coefficient and dynamic contrastenhanced MR imaging-derived measurement of the volume of the extravascular extracellular space in glioblastoma multiforme. Am. J. Neuroradiol. 2010; 31 (3): 549-553. DOI: 10.3174/ajnr.A1844.

28. Zhang N., Zhang L., Qiu B., Meng L., Wang X., Hou B.L.Correlation of volume transfer coefficient Ktrans with histopathologic grades of gliomas. J. Magn. Reson. Imaging. 2012; 36 (2): 355-363.DOI: 10.1002/jmri.23675. 